\title{
The assessment of management of strabismus and amblyopia and national audit by Wickham
}

Eye (2002) 16, 505-506. doi:10.1038/

sj.eye. 6700249

The management of children with amblyopia and strabismus is controversial. There are questions with regard to screening, should it be at three or five years of age, who should do it and using which test of visual function. Who should carry out the diagnosis and who should do the follow-up and management and for how long?

Aside from the question of whether or not treatment works, ${ }^{1}$ there are many instances where treatment fails and several studies document poor compliance with occlusion regimes. ${ }^{2}$ Wickham $e t a l^{3}$ have described a snapshot of management of strabismus and amblyopia in the UK. Practice varies widely, constrained by staff availability, funding and geography but also tradition and habit. The authors have identified areas of weak practice such as high rates of follow-up for clinically normal children. In some areas such as the use of $\log$ MAR charts, there is supportive evidence which is being ignored but in many areas evidence, such as the choice of the optimum test for visual acuity at different ages and whether management by an orthoptist and optometrist team, including fundoscopy and refraction, is both safe and cost effective, is simply not available to justify practice. It is helpful that the authors have described their own protocol as this can provide a useful starting point for debate but it would be useful also to have more detailed guidelines from one of the professional bodies to outline best practice.

As the authors say, many papers have shown that LogMAR-based tests are superior
RA Harrad and C Williams

for all acuity testing, especially in children where the old-fashioned alternatives are comparatively insensitive. For children too young to be able to manage LogMAR tests (ie below 2.5 or 3 years in some cases), however, it might be preferable to increase awareness of the weaknesses of existing tests so that their results are applied properly, rather than abandoning all forms of VA testing in toddlers, as some useful information (medium to gross disparity between the eyes, development of VA with age or treatment) can still be gained from PL or Cardiff Cards, beyond that given by 'objection to occlusion'. This is not to say that VA tested by these less reliable methods in younger $(<2.5$ years) children should be a reason for follow-up in the absence of a family history of strabismus or any refractive error, as the risk of subsequent strabismus or amblyopia for such children is very low. Firm data to quantify this risk would be useful, not least in order to advise and inform parents.

The confidence with which a child may be said to be too young to have their vision tested reliably also depends to some extent on what 'safety net' is available to detect problems later. The UK National Screening Committee's interim recommendations ${ }^{4}$ that all children should have a vision check by an orthoptist between the ages of 4 and 5 acknowledge the importance of appropriate training and standards for vision testing. Such a programme could provide an ideal opportunity for the piloting and establishment of national standards for vision testing, referral and management protocols for children of this age with strabismus and amblyopia, with subsequent 'working backwards' towards agreement for younger
Bristol Eye Hospital Lower Maudlin St Bristol BS1 2LX, UK

Correspondence: RA Harrad

Tel: 01179284689

Fax: 01179284686 bristol.ac.uk E-mail:R.A.Harrad@ 
children. The relevant Colleges are ideally placed to lead the way in this. Wickham et al's paper provides a useful overview of the current variability in practice and highlights this challenge to paediatric ophthalmology.

\section{References}

1 Snowden S, Stewart-Brown S. Pre-school vision screening: results of a systemic review. NHS Centre for Reviews and Dissemination, University of York, 1997.
2 Searle A, Vedhara K, Norman P, Frost A, Harrad R. Compliance and eye patching in children and its psychosocial effects: a qualitative application of protection motivation theory. Psychol Health Med 2000; 5: 43-54.

3 Wickham L, Stewart C, Charnock A, Fielder A. The assessment and management of strabismus and amblyopia: a national audit. Eye 2002; 16: 522-529.

4 Rahi J, Williams C, Bedford H, Elliman D. Screening and surveillance for ophthalmic disorders and visual deficits in children in the United Kingdom. Br J Ophthalmol 2001; 85: 257-259. 